\title{
No enhancement of the localization length for two interacting particles in a random potential
}

\author{
Rudolf A. Römer and Michael Schreiber \\ Institut für Physik, Technische Universität Chemnitz, D-09107 Chemnitz, Germany \\ (Version: October 24, 1996; printed December 3, 1996)
}

\begin{abstract}
We study two interacting particles in a random potential chain by means of the transfer matrix method. The dependence of the two-particle localization length $\lambda_{2}$ on disorder and interaction strength is investigated. Our results demonstrate that the recently proposed enhancement of $\lambda_{2}$ as compared to the results for single particles is entirely due to the finite size of the systems considered. This is shown for a Hubbard-like onsite interaction and also a long-range interaction.
\end{abstract}

71.55.Jv, 72.15.Rn, 72.10.Bg

In one dimension (1D), bosonization of the repulsive Hubbard model in the gapless phase shows that (onsite) disorder is a relevant perturbation and the ground state corresponds to a strongly localized phase [1]. Thus it came as a surprise, when Shepelyansky [2] recently argued that the Hubbard interaction between two particles in a random potential would reduce the localization in comparison with independent particles. In particular, he obtained an enhancement of the two-particle localization length $\lambda_{2}$ independent of the statistics of the particles and of the sign of the interaction such that

$$
\lambda_{2} \approx U^{2} \frac{\lambda_{1}^{2}}{32}
$$

in the band center. Here $\lambda_{1}$ is the single particle localization length in $1 \mathrm{D}$ and $U$ the Hubbard interaction in units of the nearest-neighbor hopping strength. Shepelyansky obtained this result by studying the matrix representation $\mathbf{U}$ of the Hubbard interaction in the disorderdiagonal basis of localized single particle eigenstates, i.e., $\mathbf{U}=U Q_{n m, k l}=U \sum_{i} \phi_{n}^{\dagger}(i) \phi_{m}^{\dagger}(i) \phi_{k}(i) \phi_{l}(i)$, with $\phi_{n}(i)$ the amplitude at site $i$ of the single particle eigenstate with energy $E_{n}$. Here, he assumed that $\phi_{n}(i)$ behaves as $\phi_{n}(i) \sim r_{n} / \sqrt{\lambda_{1}}$, where $r_{n}$ is a random number of order unity, and he neglected correlations among the $r_{n}$ at different sites resulting in a Gaussian distribution for the matrix elements of $Q$.

Support for this result was given shortly afterwards by Imry [3] with the help of a Thouless-type scaling argument. Frahm et al. [4] used the transfer matrix method (TMM) to study the two-particle Hubbard-Anderson problem without any approximations and have found numerically that $\lambda_{2} \sim \lambda_{1}^{1.65}$. They also measured the distribution of the matrix elements of $Q$ and found a strongly non-Gaussian behavior. They attribute the deviation of the exponent from Eq. (1) and the different distribution of matrix elements to the correlations among the $r_{n}$ neglected by Shepelyansky. Subsequently, an approximate calculation of $\lambda_{2}$ by Oppen et al. [5] with Green function methods lead to the hypothesis $\lambda_{2}=\lambda_{1}+C \frac{|U|}{\pi} \lambda_{1}^{2}$, with $C \approx 0.34$ for bosons and 0.36 for fermions. Oppen et al. also identified a scaling parameter $U \lambda_{1}$. Recently, Weinmann et al. [6] argued that for a Gaussian matrix ensemble there exists a crossover from the $|U|$ behavior to the $U^{2}$ behavior for increasing $U$, but with a different exponent, such that $\lambda_{2}=\lambda_{1}+A \frac{|U|}{4+W} \lambda_{1}^{3 / 2}$ for small $U$ with $W$ parametrizing the disorder.

We feel that in this ongoing controversy about the interaction and disorder dependence, the system size has not been appropriately taken into consideration. We doubt the existence of the enhancement for an infinite system size, because there is only a vanishing probability that two localized particles are sufficiently close on an infinite chain to "see" each other. To substantiate this argument, we present in this Letter extensive computations of $\lambda_{2}$ in dependence of the system size $M$. We first review the TMM approach to the two interacting particles (TIP) problem and suggest an improved TMM based on the self-averaging properties of the Lyapunov exponents. With this method we reproduce the $\lambda_{2}$ estimates of Ref. [4] for $U=1$, system size $M=100$, and all disorders. We find (cp. Fig. 1), however, that the enhancement $\lambda_{2} / \lambda_{1}$ decreases with increasing $M$. We also study the behavior of $\lambda_{2}$ for $U=0$ and show in Fig. 1 that it is not equal to $\lambda_{1}$ for finite $M$. But we recover the $\lambda_{1}$ value [7] in the limit $M \rightarrow \infty$. However, the enhancement $\lambda_{2} / \lambda_{1}$ also vanishes completely in this limit as demonstrated in Fig. 1.

The Schrödinger equation for the TIP problem with Hubbard interaction is written in a suggestive form as

$$
\begin{aligned}
\psi_{n+1, m}= & {\left[E-\left(\epsilon_{n}+\epsilon_{m}\right)-U \delta_{n, m}\right] \psi_{n, m} } \\
& -\psi_{n, m+1}-\psi_{n, m-1}-\psi_{n-1, m},
\end{aligned}
$$

where $n, m=1, \ldots, M$ are the two site indices of the particles, $E$ is the total energy of both particles, and $\epsilon_{i}$ is the random potential at site $i$. In the following, we use a box distribution $[-W / 2, W / 2]$ for the $\epsilon_{i}$. The single particle localization length in $1 \mathrm{D}$ for such a distribution is known [8] from second order perturbation theory in $W$ to vary with disorder and energy as $\lambda_{1} \approx 24\left(4-E^{2}\right) / W^{2}$. The numerical results for $E=0$ based on the TMM [7] lead to a slightly different prefactor

$$
\lambda_{1} \approx 105 / W^{2}
$$

If one interprets $(n, m)$ as Cartesian coordinates on a finite lattice with $M \times M$ sites, the problem becomes 
identical to a non-interacting Anderson model in 2D with disorder potential symmetric with respect to the diagonal $n=m$ and with hard wall boundary conditions [4,9]. One can rewrite Eq. (2) in the TMM form as

$$
\left(\begin{array}{l}
\psi_{n+1} \\
\psi_{n}
\end{array}\right)=T_{n}\left(\begin{array}{l}
\psi_{n} \\
\psi_{n-1}
\end{array}\right)
$$

with the symplectic transfer matrix

$$
T_{n}=\left(\begin{array}{cc}
E \mathbb{1}-\chi_{n}-H_{-} & -\mathbb{1} \\
\mathbb{1} & \mathbf{0}
\end{array}\right)
$$

where $\psi_{n}=\left(\psi_{n, 1}, \ldots, \psi_{n, m}, \ldots, \psi_{n, M}\right)$ is the wave vector of slice $n, H_{-}$is the single-particle hopping term for the transverse $(m)$ electron and $\left(\chi_{n}\right)_{i, m}=\left(\epsilon_{n}+\epsilon_{m}+\right.$ $\left.U \delta_{n, m}\right) \delta_{i, m}$ codes the random potentials and the Hubbard interaction [4]. Note that in this approach the symmetry of the wave function remains unspecified and we cannot distinguish between boson and fermion statistics.

The evolution of the state is determined by the matrix product $\tau_{N}=\prod_{n=1}^{N} T_{n}$ and we have

$$
\left(\begin{array}{l}
\psi_{N+1} \\
\psi_{N}
\end{array}\right)=\tau_{N}\left(\begin{array}{l}
\psi_{1} \\
\psi_{0}
\end{array}\right)
$$

Usually, the method is performed with a complete and orthonormal set of initial vectors $\left(\psi_{1}, \psi_{0}\right)^{T}$. The eigenvalues $\exp \left[2 \gamma_{i}(N)\right]$ of $\left(\tau_{N}^{\dagger} \tau_{N}\right)^{1 / N}$ exist for $N \rightarrow \infty$ due to Oseledec's theorem [10]. The smallest Lyapunov exponent $\gamma_{\text {min }}$ determines the slowest possible decay of the wave function and thus the largest localization length $\lambda_{\max }=1 / \gamma_{\min }$. We now define the two-particle localization length $\lambda_{2}$ as $\lambda_{\text {max }}$ of the transfer matrix problem (6).

In the TMM studies of the Anderson Hamiltonian, one usually multiplies the transfer matrices in the $n$ direction until convergence is achieved, e.g., for a $2 \mathrm{D}$ sample with strip width $M=100$ and $W=2$, this typically requires $N=5 \times 10^{6}$ multiplications for an accuracy of $1 \%$. Thus one effectively studies a quasi-1D system of size $M \times N$ with $M \ll N$. However, in the present problem, both directions are restricted to $n, m \leq M$. Iterating Eq. (4) only $M$ times will not give convergence. Frahm et al. [4] have solved this problem in their TMM study by exploiting the hermiticity of the product matrix $Q_{M}=\tau_{M}^{\dagger} \tau_{M}$ : Continuing the iteration (6) with $\tau_{M}^{\dagger}$, then with $\tau_{M}$, and so on, until convergence is achieved, yields the eigenvalues $\exp \left[2 \gamma_{i}(N)\right]$ of $\left(\prod^{N / M} Q_{M}\right)^{1 / N}$. This means that they repeat the same $M \times M$ sample $N / M$ times. For $M=100$ and $W=2$ we found that $N / M \approx 5000$ is necessary to give an accuracy of $5 \%$. Since this has to be done for every disorder configuration, the computational effort is quite large. However, in the usual quasi1D TMM for single particles, one may also halt the matrix multiplication every $L \ll N$ steps, compute an estimate for $\gamma(L)$ and finally average over all $N / L$ such values [11]. Due to the self-averaging property of the
Lyapunov exponents [12] the averaged localization length equals the converged localization length $\lambda(N)$ within the accuracy of the calculation. This suggests the following method: We iterate $\prod_{r=1}^{N / M} \tau_{r, M}$, where $r$ enumerates different samples of size $M \times M$. Next we compute a localization length $\lambda(N)$ which is now already averaged over $N / M$ different samples. This significantly reduces the computational effort and allows us to study much larger systems and achieve a significantly better accuracy than previously.

In Fig. 2 we show the results obtained for the localization length of the TIP problem with Hubbard interaction by the present TMM. The data is calculated by configurationally averaging at least 10000 samples for each data point. This corresponds to an accuracy of $1 \%$ for the smallest $W$ considered and better for larger $W$. We note that the data for $U=1$ agree well with the results obtained in Ref. [4] for $1.4 \leq W \leq 4$ [13] and we find $\lambda_{2} \sim W^{-3.8}$. Furthermore, for $W \bar{\epsilon}[1.4,5]$, the data can also be fitted reasonably well by

$$
\lambda_{2}=\lambda_{1}+A \lambda_{1}^{\alpha} /(B+W)
$$

with $\alpha=2$ with fit parameters $A=0.37$ and $B=0.7$. A fit with $\alpha=3 / 2$ according to Ref. [6] is considerably worse and without the denominator in Eq. (7), as suggested in Ref. [5], the data cannot be fitted at all. We emphasize that the TIP data differ significantly from the 2D TMM data with uncorrelated 2D random potential $\epsilon_{n, m}$.

In Fig. 2 we have also plotted the behavior for $U=0$, where the system reduces to two non-interacting particles in 1D. Before analyzing the data, let us first state our expectations: For $U=0$, the two particles will localize independently at two arbitrary sites, say $n_{0}$, $m_{0}$, with localization length $\lambda_{1}$. The wave function can then be written as a product of two exponentially decaying single particle wave functions, i.e. $\psi_{n, m}^{(0)} \sim$ $\exp \left[-\left|n-n_{0}\right| / \lambda_{1}\left(E_{0}\right)\right] \exp \left[-\left|m-m_{0}\right| / \lambda_{1}\left(-E_{0}\right)\right]$. Here, the eigenenergies are chosen such that $E=0$. Two points are worth mentioning: (i) Let $\gamma_{1}\left(E_{\alpha}\right)$ denote the inverse decay length measured by TMM for a single particle with energy $E_{\alpha}$ and $\gamma_{2}(0)$ the inverse decay length for two particles with $E=0$. For $U=0$, the TMM for two particles will measure an inverse decay length $\gamma_{2}(0) \approx \sum_{\alpha}^{\mathcal{N}} \gamma_{1}\left(E_{\alpha}\right) / \mathcal{N}$, averaged over $\mathcal{N}$ pairs of states with energies $E_{\alpha}$ and $-E_{\alpha}$. Since $\gamma_{1}(E \neq 0)>\gamma_{1}(0)$ as mentioned above Eq. (3), we have $\gamma_{2}(0)>\gamma_{1}(0)$ and consequently $\lambda_{2}(0)<\lambda_{1}(0)$. (ii) The non-interacting two-particle wave function $\psi_{n, m}^{(0)}$ is not isotropic in the 2D plane $(n, m)$. Since the TMM will not necessarily measure the decay directly in the $n$ direction, we expect that $\gamma_{2}$ will also contain information about the decay in other directions. These decay lengths of $\psi_{n, m}^{(0)}$ are shorter and thus we again expect $\lambda_{2}(0) \leq \lambda_{1}(0)$.

Figure 2 shows that contrary to our expectations the TMM does not under- but overestimate $\lambda_{2}$ as compared to $\lambda_{1}$ such that the enhancement $\lambda_{2}(U=1) / \lambda_{2}(U=0)$ is 
much smaller than the previously reported $\lambda_{2}(U=1) / \lambda_{1}$. Also included in Fig. 2 is a $1 \mathrm{D}$ TMM result and we see that for $W \geq 6$ - where the perturbative result (3) is no longer accurate - both the $U=0$ and the $U=1$ data agree quite well with $\lambda_{1}$. For $W \in[1.4,4]$ a power law fit gives $\lambda_{2}(U=0) \sim W^{-3.6}$. A fit with $\alpha=2$ as in Eq. (7) can also describe the data for small $W$ with $A=0.19$ and $B=0.5$.

We emphasize that the deviation from Eq. (3) of our data for vanishing $U$ does not result from an effective coupling which was suspected [14] to be introduced into the problem because of numerical instabilities of the TMM. Our tests did not show such a coupling. We also note that the two-particle TMM data is quite different from data of a $2 \mathrm{D}$ single-particle calculation for $M=100$ as shown in Fig. 2.

Studying the $U$ dependence of $\lambda_{2}$, we note that $\lambda_{2}(U)=\lambda_{2}(-U)$ at $E=0$ due to particle-hole symmetry. Consequently, we only show data for $U \geq 0$ in Fig. 3. We observe neither the proposed $U^{2}$ nor the $|U|$ behavior. Rather the data obey $\lambda_{2}(U)-\lambda_{2}(0) \approx U^{0.63}$ for small $U$. Also, $\lambda_{2}(0) \approx 8.45$ does not agree with $\lambda_{1} \approx 105 / 16$, as discussed above, and we cannot identify a scaling parameter as suggested in Ref. [5].

From Fig. 2 we see that for $W>6$, the data for $U=0$ and $U=1$ coincide with the single-particle TMM data already quite well. The enhancement $\lambda_{2} / \lambda_{1}$ is large only for small disorder $(W \leq 4)$. However, in this region, the computed values of $\lambda_{2}$ become comparable to the size of the system such that an increasing part of the wave function is reflected at the hard wall boundaries. This leads to an overestimation of $\lambda_{2}$. In order to distinguish this artificial from the proposed enhancement due to interaction alone, we have therefore systematically studied the finite-size behavior of $\lambda_{2}$ at various disorder values and interaction strengths. As an example, we consider the case $W=3$. Here the localization lengths for $\lambda_{2}(U=0) \approx 19.1$ and $\lambda_{2}(U=1) \approx 25.5$ may just be small enough for the wave function to fit into the $100 \times 100$ box without too strong boundary effects. Furthermore, the enhancement is still sufficiently large to be numerically detectable. In Fig. 1, we have shown $\lambda_{2}$ as a function of $M^{-1 / 2}$. Our reason for plotting the data as a function of $M^{-1 / 2}$ is pragmatic: a plot versus $M^{-1}$ or $M^{-1 / 4}$ shows a downward or upward curvature, respectively, for the largest $M$. The curvature is smallest for $M^{-1 / 2}$ and the extrapolation towards infinite $M$ is most reliable. We see in Fig. 1 that (i) the data for $U=0$ approach the single-particle result $\lambda_{1} \approx 105 / 9$ for large $M$, and, more significantly, (ii) the data for $U=1$ also approach this non-interacting $1 \mathrm{D}$ result. Therefore the overestimation of $\lambda_{2}(U=0)$ vanishes for large $M$, supporting the validity of the TMM approach. However, in this limit the enhancement $\lambda_{2}(U=1) / \lambda_{1}$ vanishes completely, too. We note that the observed decrease in the localization length $\lambda_{2}$ is quite different from a 2D TMM, where the localization length is known to increase with increasing system size [9]. We attribute this difference to the symmetry of the TIP disorder potential which is equivalent to an effective long-range correlation which furthermore increases with increasing $M$.

We have also studied the behavior of the two-particle localization length for a system with long-range interaction, since Shepelyansky argues in Ref. [15] that longrange interaction should also lead to the enhancement of $\lambda_{2}$. The Schrödinger equation is given as

$$
\begin{gathered}
\psi_{n+1, m}=\left[E-\left(\epsilon_{n}+\epsilon_{m}\right)-U /(|n-m|+1)\right] \psi_{n, m} \\
-\psi_{n, m+1}-\psi_{n, m-1}-\psi_{n-1, m}
\end{gathered}
$$

such that the on-site interaction is equal to the Hubbard interaction. Clearly such a system should exhibit even stronger boundary effects and we thus expect an even stronger enhancement of $\lambda_{2}$ for finite systems. In Fig. 1 , we have also shown $\lambda_{2}$ for the long-range interaction with $U=1$ at $E=0$ and the same accuracy of $1 \%$ as for the Hubbard interacting system. As expected, we observe that the enhancement for all $M$ is stronger than for the Hubbard interacting model. But this enhancement again vanishes for $M \rightarrow \infty$.

In summary, we have studied the interaction-induced enhancement of the localization length for two particles in a $1 \mathrm{D}$ random potential by means of an improved TMM. For $U=1$ and $M=100$, the results agree well with previously published data. However, the $U=0$ data is quite surprising since only in the limit $M \rightarrow \infty$ do we recover the expected non-interacting $1 \mathrm{D}$ result $\lambda_{1}$. However, in this limit the enhancement for finite $U$ also vanishes. Therefore we must conclude that the transfer matrix method applied to the 1D Anderson model for two interacting particles measures an enhancement of the localization length which is entirely due to the finiteness of the systems considered. This enhancement might be relevant for applications in mesoscopic systems.

\section{ACKNOWLEDGMENTS}

We thank Frank Milde for programming help and discussions. This work has been supported by the Deutsche Forschungsgemeinschaft as part of Sonderforschungsbereich 393 .

[1] T. Giamarchi and H. J. Schulz, Phys. Rev. B 37, 325 (1988).

[2] D. L. Shepelyansky, Phys. Rev. Lett. 73, 2607 (1994).

[3] Y. Imry, Europhys. Lett. 30, 405 (1995).

[4] K. Frahm, A. Müller-Groeling, J. L. Pichard and D. Weinmann, Europhys. Lett. 31, 169 (1995).

[5] F. v. Oppen, T. Wettig and J. Müller, Phys. Rev. Lett. 76, 491 (1996). 
[6] D. Weinmann and J. L. Pichard, preprint (1996).

[7] G. Czycholl, B. Kramer and A. MacKinnon Z. Phys. B 43, 5 (1981); J. L. Pichard, J. Phys. C 19, 1519 (1986).

[8] D. J. Thouless, J. Phys. C 5, 77 (1972).

[9] A. MacKinnon and B. Kramer, Z. Phys. B 53, 1 (1983).

[10] V. I. Oseledec, Trans. Moscow Math. Soc. 19, 197 (1968).

[11] B. Kramer and M. Schreiber, in Computational Physics, eds. K. H. Hoffmann and M. Schreiber (Springer, Berlin, 1996), p. 166.

[12] J. Sak and B. Kramer, Phys. Rev. B 24, 1761 (1981); K. S. Chase and A. MacKinnon, J. Phys. C 20, 6189 (1987); M. Schreiber and B. Kramer, in The Physics of Semiconductors, ed. W. Zawadski (Institute of Physics, Polish Academy of Sciences, Warsaw, 1988), p. 87; B. Kramer and M. Schreiber, J. Non-Cryst. Sol. 114, 330 (1989).

[13] Note that in Ref. [4] the disorder distribution $[-W, W]$ is used.

[14] A. Müller-Groeling, (private communication).

[15] D. L. Shepelyansky, preprint (1996).

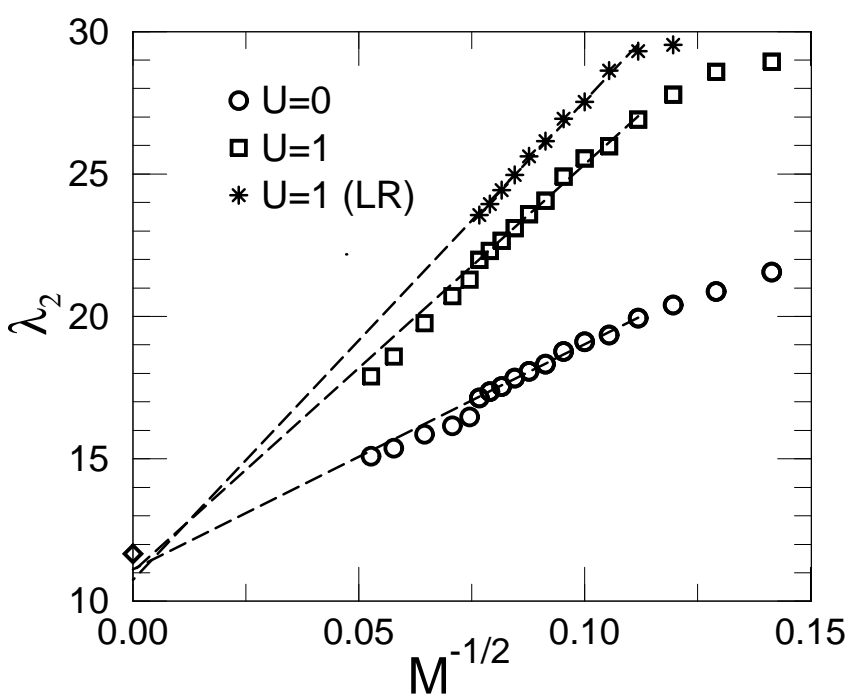

FIG. 1. Two-particle localization length $\lambda_{2}$ as a function of the system size at $W=3$ for Hubbard (2) and long-range interacting systems $(*)$. Dashed lines are extrapolations from the data for $M \in[80,170]$. The 5 leftmost data points for $U=0$ and Hubbard $U=1$ corresponding to $M=180,200,240,300,360$ have been computed with only $5 \%$ accuracy and have not been considereded for the extrapolations. The diamond $(\diamond)$ indicates the value of $\lambda_{1}$.

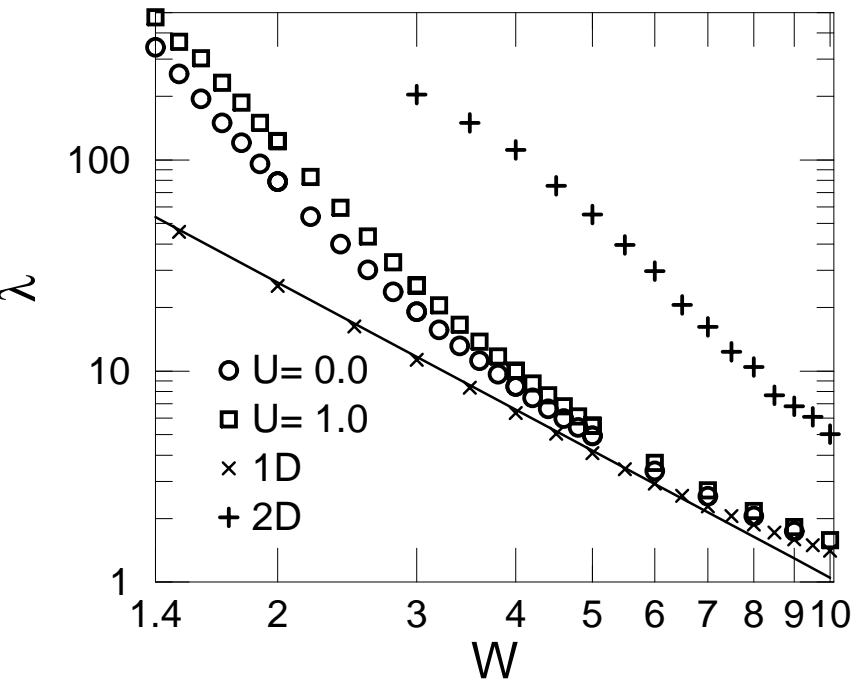

FIG. 2. Two-particle localization length $\lambda_{2}$ (accuracy at least $1 \%$ ) at energy $E=0$ for system size $M=100$ and interaction strength $U=0$ (o) and 1 (2). The solid line is given by Eq. (3). Single-particle TMM data with 1\% accuracy for $1 D$ chains $(x)$ and $2 \mathrm{D}$ strips of width $M=100(+)$ are also shown.

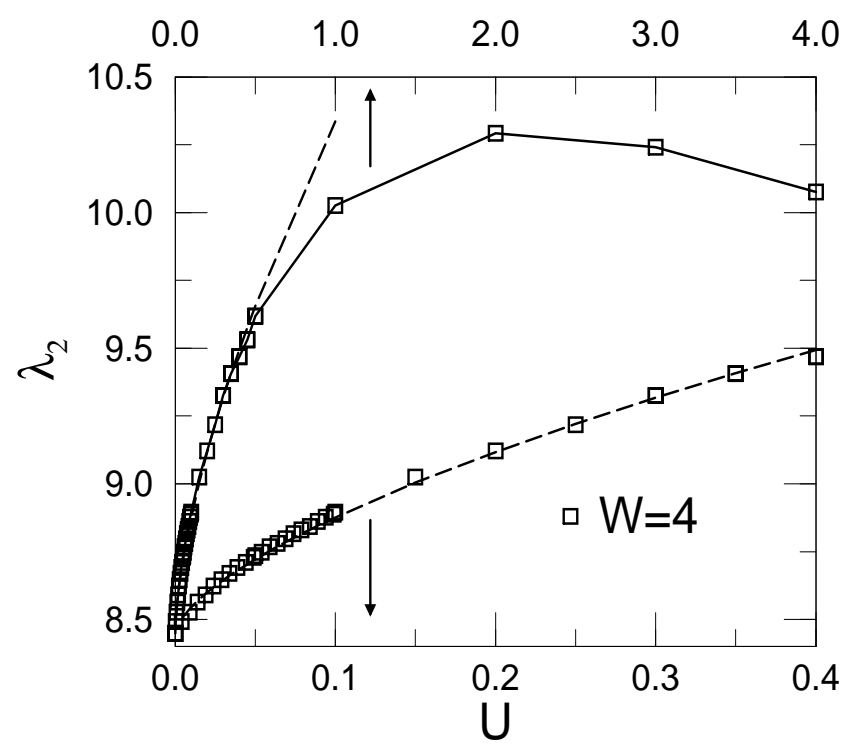

FIG. 3. $U$ dependence of $\lambda_{2}$ for $W=4$. The dashed line represents the power law fit $\lambda_{2}(U)-\lambda_{2}(0) \approx U^{0.63}$. The lower curve shows the same data on an enlarged $U$ scale. Arrows indicate the corresponding axes. 